\title{
RoboCup MSL - History, Accomplishments, Current Status and Challenges Ahead
}

\author{
Robin Soetens $^{1(\otimes)}$, René van de Molengraft ${ }^{1}$, and Bernardo Cunha ${ }^{2}$ \\ 1 Eindhoven University of Technology, Eindhoven, The Netherlands \\ robinsoetens@gmail.com \\ ${ }^{2}$ IRIS Lab/IEETA/DETI, University of Aveiro, Aveiro, Portugal
}

\begin{abstract}
The RoboCup Middle-Size League (MSL) is one of the founding leagues of the annual RoboCup competition. Ever since its birth it has been a league where development of hard- and software happens simultaneously in a real-world decentralized multi-robot soccer setting. Over the years the MSL achieved scientific results in robust design of mechatronic systems, sensor-fusion, tracking, world modelling and distributed multi-agent coordination. Because of recent rule changes which actively stimulate passing, matches in RoboCup MSL have become increasingly appealing to a general audience. Approximately five thousand spectators were present during last years final match. In this paper we present our plan to build on this momentum to further boost scientific progress and to attract new teams to the league. We also give a historical overview and discuss the current state of the MSL competition in terms of strengths, weaknesses, opportunities and threats.
\end{abstract}

Keywords: RoboCup $\cdot$ Middle-size league $\cdot$ Roadmap $\cdot$ History $\cdot$ Academic competition $\cdot$ Open source $\cdot$ Survey $\cdot$ Outlook

\section{Introduction}

Picture two teams of five robotic players each, playing with an official FIFA ball on an indoor field. The team in possession of the ball coordinates passes and dribbles to bring the defending robots out of position, eventually they find a weak spot, score, and win. Perception is done by on-board sensors only, there is no centralized vision system. Robots communicate and build a world model based on information coming from itself and from team mates. No human intervention is allowed, except for the referee and a dedicated human coach who is allowed to provide high-level strategic instructions (but only during dead-time and only by showing signs, making gestures, or shouting).

Our perception, as spectators, brings us into the thrill of watching a real football game. The heart beats faster and we are anticipating the next best move, or we are plunging into a feeling of frustration by the missed opportunity or the incomprehensible action of a specific player. The sensations follow up and down at the rhythm and dynamics of the game. You find yourself yelling "shoot" or "pass," while mapping human emotions to your favourite robot football team.

(C) Springer International Publishing Switzerland 2015

R.A.C. Bianchi et al. (Eds.): RoboCup 2014, LNAI 8992, pp. 624-635, 2015.

DOI: $10.1007 / 978-3-319-18615-3 \_51$ 
This is what a RoboCup MSL game unveils to those who watch it live. Players move around on wheels, are short and gross while lacking arms and legs. They are $80 \mathrm{~cm}$ tall, reach speeds of $60 \%$ to $70 \%$ of an average human top speed and weight up to $40 \mathrm{~kg}$ each. Those who watch them for the first time suddenly realize that "Yes, they are fully autonomous, they play soccer all by their own." The same feeling also arises for those who, on the other side of the chain, are working towards a solution for one of hundreds of challenges still to be tackled.

These are not yet, for sure, robots that have fulfilled the dream stated by the RoboCup Federation as "By 2050, a team of fully autonomous humanoid robot soccer players shall win a soccer game, complying with the official FIFA rules, against the winner of the most recent world cup." But, at this time, in the path towards that goal, they rise the imagination of a spectator to a level where a real soccer game can be seen beyond the science and technology hidden inside.

\subsection{Relevance to Society}

Since its first tournament in 1997, the RoboCup competition boosts both research and development of technology in artificial intelligence and robotics. At the same time the appealing nature of doing science by playing soccer also inspires young people and promotes science and technology among a general audience. It has potential to attract thousands of spectators to watch and cheer for a team of robots (Fig. 1). For participants, the element of competition brings up additional energy. A RoboCup tournament provides an open atmosphere, which facilitates knowledge exchange. It allows to acquire hands-on experience with real-world systems, while working under high pressure of time in a team with experts in different domains. RoboCup experience is a tremendous addition to the training of an engineer.

Boosting research, promoting science and training future engineers are three pillars of societal relevance that justify the allocation of resources required to organize a RoboCup competition. MSL contributes to each of these pillars. It boosts research by providing a real-world multi-robot benchmark involving real hardware and the disturbances and constraints that come with a real game of soccer. It promotes science and technology because its format is easy to grasp and explain to a general audience and to media. And since teams typically consist of ten to fifteen people, with a mix of experienced and less experienced team members, MSL is an ideal cradle for education and training of future engineers.

\subsection{Organization}

The remainder of this paper is organized in three sections: First we provide an historical perspective on evolution and revolution we have seen in MSL. Next we present the current status of the league and elaborate on how challenges are being addressed. Lastly, in the final section, we look at the future and put efforts being made today in the perspective of a long-term goal. Short conclusions and a link to a reference digest conclude the article. 


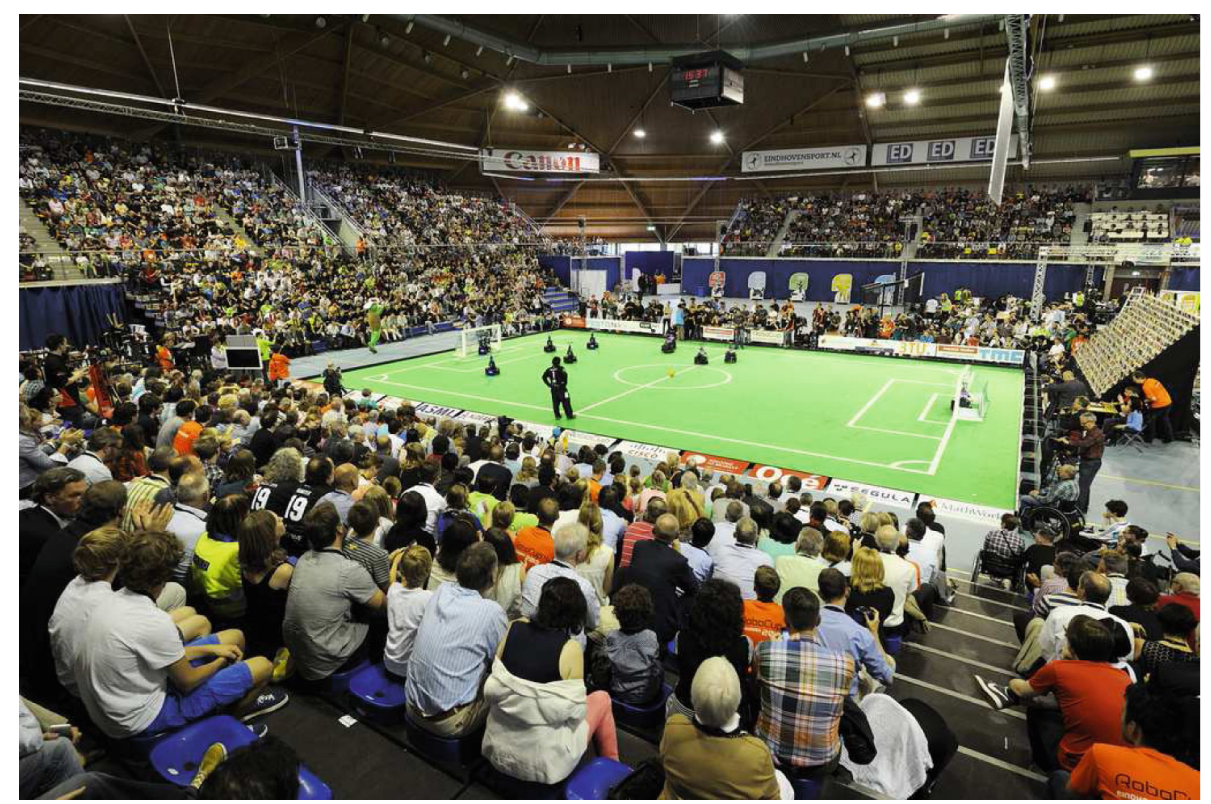

(a) Over 5000 spectators during the final match of RoboCup 2013 in Eindhoven.

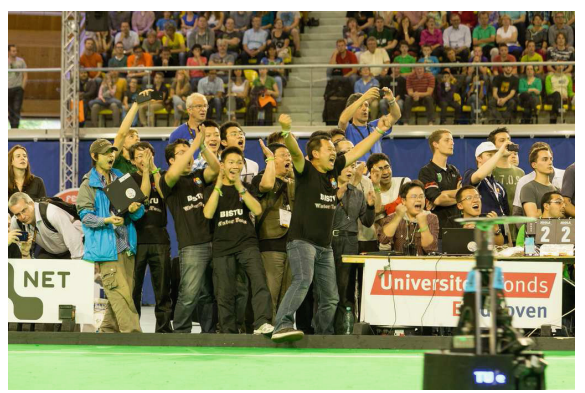

(b) Team Water from the Beijing Information Science and Technology University after winning RoboCup 2013.

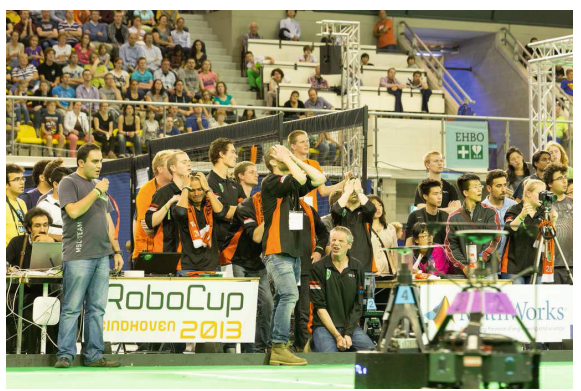

(c) Team Tech United from Eindhoven University of Technology after losing the final match of RoboCup 2013.

Fig. 1. Public engagement and team emotions in RoboCup MSL. Photographers: Bart van Overbeeke (a), Albert van Breemen (b) and (c)

\section{The Past}

Although neither of the authors of this paper participated in the league from the beginning, from a scientific and a technical point of view it is possible to identify a number of well-constrained epochs, each representing a period of time where development was established through evolution towards a certain challenge. Epoch boundaries, on the other hand, represent instants where development was driven by a leap step, aiming new, more daring, scientific targets. 

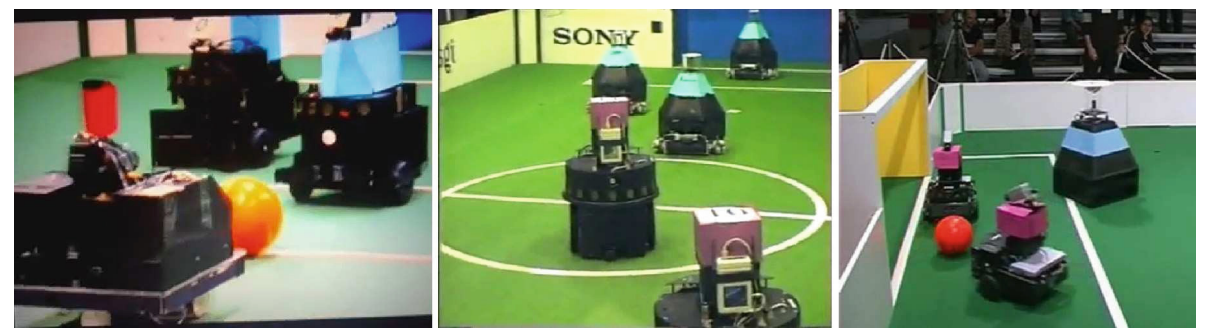

Fig. 2. From left to right: Sharif CE (Iran) vs. Azzurra Robot Team (Italy) - 1999; Golem Team (Mexico) vs. COPS (Germany) - 2000; CS-Freiburg (Germany) vs. RoboSix (France) - 2000 (Color figure online)

Throughout the years, this evolution versus revolution approach was balanced based on the ability of the community to evolve and share the produced knowledge. Powerful tools to achieve this balance include the annual updates to the MSL rulebook and the technical challenge competition, held at every RoboCup final tournament. A short overview of each of the epochs is presented next.

First Epoch: 1997 Through 2001. This was the starting up period. Original field dimensions were $9 \times 5 \mathrm{~m}$, limited by surrounding walls that kept the ball always inside the field (Fig. 2). Goals were color coded (yellow and blue) and the ball was bright orange. The rules were very simple, although already FIFA based. Teams played with up to four robots, and start and stop commands could be issued through a wireless connection by each team.

Illumination was artificial and uniform with variations below 300 lux. Teams were allowed to reposition the robots manually throughout the game. Research was centred on basic navigation and vision, mainly focussing on color-based classification and detection of objects. Speed was typically below $1 \mathrm{~m} / \mathrm{s}$. Differential traction solutions and simple electro-mechanical kickers were the focus at the mechatronics level, where solenoid-based kickers are starting to be used (e.g., at the Minho team). The first omnidirectional solutions, both at vision and traction level, start appearing around 2000.

Second Epoch: 2002 Through 2006. In the first year of this epoch the outside walls of the field were removed. Instead, color coded posts were placed on the four corners of the field. Manual repositioning of the robots was no longer allowed, except for a kick-off. Also, to simplify the game control, robots were supposed to stop if the ball was removed from the ground. Base colors were kept untouched as well as uniform artificial illumination over the field. Starting in 2004, the size of the field was increased to $12 \times 8 \mathrm{~m}$, now including both a penalty area and a goal area. In 2005, the first version of the referee box was introduced to allow a team-independent control of the game flux. Team description papers became mandatory in 2004. Common themes were real-time 
adaptive color segmentation, stronger and more precise kicking devices based on pneumatic or solenoid actuation, solutions for catadioptric vision systems, efficient omnidirectional driving, open loop dribbling devices, early sensor-fusion techniques, self-localization and first solutions for team coordination.

Third Epoch: 2007. This was a transition year where the size of the field got increased to $18 \times 12 \mathrm{~m}$, which is still the current MSL official size. Artificial uniform illumination was no longer required and the first attempt to move into a more cooperative game was introduced by imposing that a goal could only be validated on the first ten seconds after a restart if touched by a second player of the team playing the restart. Posts and goals kept their original color coding but the changes boosted the necessity to increase research on efficient dynamically adjustable vision systems together with real-time coordination of the teams. Concepts as dynamic role-changes, dynamic team-formations, forceadjustable kicking systems for both lob and floor-level shots, world modelling including opponent recognition, efficient ball-tracking on the floor, path planning and distributed real-time databases for information sharing among team mates are some examples of the research tackled in this period.

Fourth Epoch: 2008 to 2011. In the beginning of this epoch the colored corner posts were finally removed and goals became white. For the first time, in a field that looks like a real soccer field, teams had to find solutions for self localization without external visual aids while also having to disambiguate the own and opponent side of the field. Also, in 2009, maximum bandwidth used by the team was limited to $20 \%$ of the IEEE $802.11 \mathrm{~b}$ standard bandwidth. In 2010, the ball no longer had to be orange, being pre-selected by the LOC. Manual repositioning of the robots was forbidden. To enforce the players ability to perform passes efficiently, all restarts imposed a minimum distance between the ball, team mates and opponents. Own players at $1 \mathrm{~m}$, opponents at $2 \mathrm{~m}$ (since 2009) and own players at $2 \mathrm{~m}$, opponents at $3 \mathrm{~m}$ (since 2011). Two additional rules were introduced in 2011, changing dramatically the dynamics of the game. On one side, goals could only be validly scored when the shot was taken within the opponent side of the field, thus reducing the run towards stronger and stronger kickers and increasing the necessity to improve game planning. At the same time, it was also imposed that while disputing the ball, only two robots, one for each team, could be in direct contact with the ball.

The new rules brought the league to a higher level of artificial intelligence and multi-agent coordination. Real-time efficient communication among robots got improved, as well as world modelling and role assignment. Other topics addressed in MSL within this period were novel solutions for active ball handling mechanisms, multi-robot coordination using set-plays, dynamic strategy changing during the game, efficient simulation tools for highly dynamic cooperation, arbitrary ball recognition, 3D ball tracking in real-time, high density data-fusion, cooperative control through objective achievement and the use of utility fields for passing. 

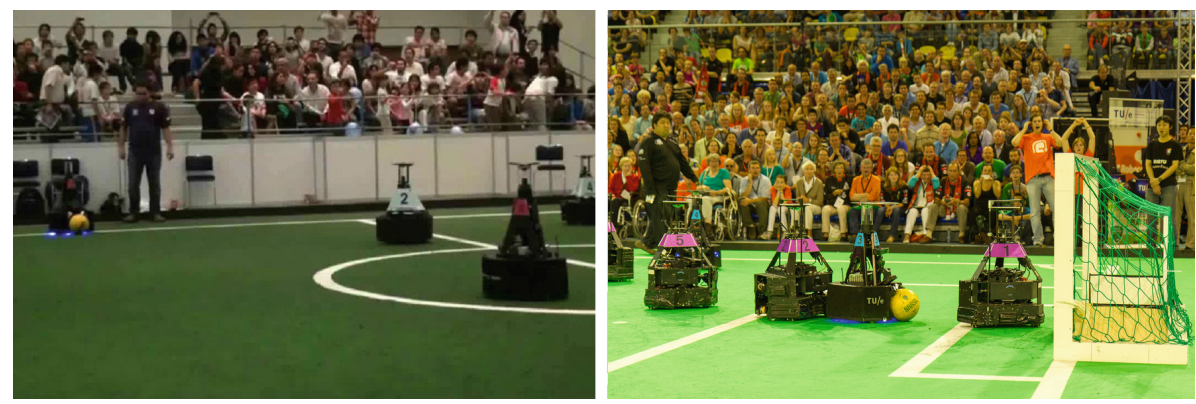

Fig. 3. Left to right: Tech United (Netherlands) vs. CAMBADA (Portugal) - 2012, Tech United (Netherlands) vs. Water (China) - 2013.

Fifth Epoch: 2012 Until Today. By 2011 it was clear that extra efforts should be put into changing dramatically the way cooperation and game play was going. Robots, at this time, were able to perform fast dribbles (between 3 and $4 \mathrm{~m} / \mathrm{s}$ ) in a controlled way, using dynamic path planning. Without the ball speeds above $4 \mathrm{~m} / \mathrm{s}$ were reached. This left the efforts of teams who were trying to exploit more cooperative solutions unrewarded, since speed became more important than strategic passing. A radical rule was introduced in 2012: Robots could no longer dribble the ball over the mid-line when progressing from their side to the opponent side. Furthermore, robots should actually make a pass to a team mate on the opponent side that could then either dribble or shoot towards the opponent goal. This counter-intuitive rule paid out. Robot average speed reduced dramatically, pushing offences became sparse, actual in-game ball passing became a reality while new strategies including man-to-man cover, zonecover or mixes of both started to appear. Active ball-interception, effective use of utility fields and passes into open zones were also thriving (Fig. 3, left).

\section{The Present}

In a contemporary RoboCup MSL match two teams of up to five robots and a base station pc play on a soccer field with an adapted version of the FIFA rules. Robots are completely autonomous (i.e., all sensors and computing equipment is on-board). They must fit in a $50 \times 50 \times 80 \mathrm{~cm}$ box and weigh no more than $40 \mathrm{~kg}$. The field is similar to a human soccer field, scaled down to $18 \times 12 \mathrm{~m}$ and with white lines over a green carpet. Goals are also scaled down.

A regular size official FIFA soccer ball is used in the tournaments. The only required human intervention comes from the referee, through a dedicated application called "referee box," which is controlled during the match by an assistant referee. Wireless networking is allowed between robots and between robots and the base station using unicast or multicast (no ad hoc or broadcast permitted). Throughout the match, consisting of two halves of $15 \mathrm{~min}$, the base station cannot use any form of sensing and cannot be touched. No permanent external active 
or passive beacons are allowed. Most teams adopt holonomic motion, based on a three or four wheel configuration, as well as omnidirectional vision, sometimes fused with panoramic and 3D vision. Ball handling can be done by active or passive devices as long as no more than a third of the ball is covered. Teams can use heterogeneous robots, where the goalkeeper, by itself, is a exceptional case, since it can have extensible parts that can momentarily extend the robot to the right, left or above, by at most $10 \mathrm{~cm}$.

For 2014, the rule prohibiting a dribble over the mid-line was dropped and replaced with a more general and natural rule: A valid goal can only be scored after the ball has been received or touched by a team mate within the opponent side of the field after rolling freely for at least one meter. Furthermore, to reduce long dribbles, a robot is now limited to dribble the ball continuously for a maximum radius of three meters with respect to the point it received the ball. The ball has to be completely released before crossing this threshold. Both of these rules enforce further cooperative and dynamic passing while the latter also encourages more dexterity in ball handling. High level human coaching by means of a QR-code is now also allowed but only during off time (e.g., when robots are positioning themselves for a free kick). In order to make progress towards playing on regular soccer fields, RoboCup 2014 will have a challenge where teams have to show basic soccer playing skills on an artificial grass field.

\subsection{Participants}

The number of participating teams in RoboCup MSL grew steadily and peaked in 2004, 2005 and 2006. For each of these tournaments the technical committee had to select a maximum of 24 teams that could participate, although more teams applied. After RoboCup Bremen in 2006, a downturn has been experienced, with only 11 teams participating in 2007, 13 in 2008 and eventually a minimum of 6 in Mexico in 2012. An average of 10 teams have participated regularly in the RoboCup main event since 2007, while nearly 30 teams stayed active and participated in regional tournaments throughout these years. ${ }^{1}$

The main reasons for the downturn after RoboCup Bremen were the high overall costs compared to other leagues, sub-optimal knowledge sharing and an international focus swing from multi-robot research to research involving a general purpose service robot. Currently, the number of participants is stable, with new teams steadily appearing and taking over the place of those who fade away. From a geographical point of view, the league is mainly represented in Europe, Asia and the Middle-East. Although research budgets in some parts of the world are a barrier, the international profile of the league would profit from new teams coming from the Americas and Africa. MSL also consistently attracts participants from industry (e.g., Philips and more recently VDL and ASML). ${ }^{2}$

\footnotetext{
${ }^{1}$ Google Maps, MSL activity over the past two years, https://goo.gl/maps/AI9aW.

${ }^{2}$ VDL, a conglomerate of manufacturing companies founded an MSL team "VDL Robot Sports" in 2012: http://www.robotsports.nl/. ASML, a lithography equipment manufacturer founded an MSL team "ASML Robo Team" in 2013.
} 


\subsection{League Profile}

Among the spectrum of different leagues, the MSL takes its own well-defined approach in its contribution to solving the RoboCup grand challenge. This approach is rooted in a number of fundamental propositions:

- Hard- and Software: In robotics, ingenious and robust design of hardware is equally important as clever software. There is as much science in developing state-of-the-art mechatronic systems as there is in advancing coordination and action. Hence, in MSL workshops and challenges we actively stimulate knowledge sharing with respect to both hard- and software design.

- Real Disturbances and Constraints: Proper benchmarking involves a real-world test where algorithms are exposed to disturbances and constraints that come with real hardware in a realistic environment. Hence MSL uses an official ball, plays on a large field, and is now focussing on playing on artificial grass instead of a smooth carpet.

- Open Mind: Progress is frequently hampered by what we know. Often we construct a robot arm that looks like a human arm because that is all we know, not because it is optimal for the task at hand. Hence, robot limitations in MSL by no means prescribe what a robot should look like. This stimulates creativity and allows taking the shortest route to FIFA compliant soccer versus a team of humans, though not necessarily with humanoid robots.

- Multi-Robot: Sharing knowledge between robots is one of the key challenges in robotics. Any general purpose service robot will have to be cooperate with other robots, or at least interact with other machinery. Hence, in MSL, we actively stimulate robot to robot cooperation and standardization of interfaces with, e.g., enforced passing and mixed team challenges.

- Focus and Integration: Robotics research groups often focus on specific topics like computer vision, motion control or machine learning. While focus is important, there is also science in integrating methods from all these fields into a single robotic system. Both approaches are possible in MSL. Teams can design and build an entire robot, but can also acquire a standardized platform (Sect. 3.3) and focus on, e.g., redesigning the vision unit.

- Semi-closed World: Deploying robots in an unstructured world still is a huge challenge. Therefore the MSL scenario is carefully placed halfway between the closed world of factories and the open world of human environments. White lines on a field of a known dimension, a preselected ball and a fixed number of ten mandatory black players provide enough structure to facilitate recognition and localisation. This allows teams to do research beyond perception, while at the same time roadmap elements like, e.g., a free choice of team shirts or playing with an arbitrary ball gradually shift the MSL scenario towards a more open world.

With the propositions above we defined MSL's focus and approach with respect to the RoboCup Grand Challenge, and with respect to other leagues. This competition profile comes with specific league properties most of which can be explained both as a strength and a weakness. In the upcoming subsections we list these and we distinguish opportunities and threats that arise from them. 

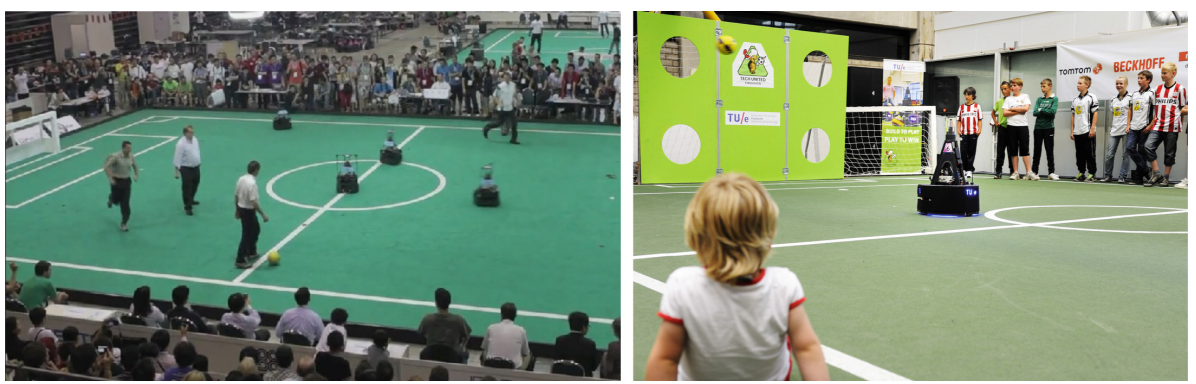

Fig. 4. Human vs robots games are played yearly since 2007 (left). MSL robot outperforms humans in shooting accuracy (right).

\section{Strengths}

- Developing hard- and software simultaneously, while facing real-world disturbances and limitations. Therefore the league is a proper benchmark test.

- High-speed game flow and easy to understand, which makes the league appealing to the general audience, to future researchers and to media.

- League with a long history, which implies lots of knowledge has been accumulated already and a large community exists.

- Highly dynamic environment with "aggressive" opponents resulted in robust hardware design and efficient real-time algorithms.

- Realistic field and ball size make the competition suitable for large numbers of spectators and allow human versus robot challenges. MSL facilitated the first ever human versus robot match in RoboCup history in 2007 and in shooting accuracy an MSL robot already beats humans (Fig. 4).

- Strong focus on robot to robot cooperation, which is important in many application domains of robotics.

\section{Weaknesses}

- Compared to other leagues, high start-up costs and time for new teams.

- High costs of participation, because teams are typically large and multiple robots have to be transported.

- League with a long history, therefore difficult for new teams to catch-up.

- Multiple robots and "aggressive" opponents implies lots of maintenance.

- Currently not all continents are represented in the MSL community.

\section{Opportunities}

- League has a long history so there is lots to document and valorize. When properly done new teams and the outside world can profit from that.

- Media and spectator friendly nature of the league makes it attractive to sponsors. Also direct participation of companies in the league can be further stimulated. In the recruiting battle for high-tech talent, companies can profit from visibility at RoboCup. 
- Real soccer dimensions, human versus robot possibilities and highly dynamic gameplay make MSL robots very useful for demoing purposes, e.g., at technology fairs or in-house days for prospect students. When properly executed, this can be an additional source of budget for teams.

\section{Threats}

- Teams quitting because of high costs.

- Difficult to keep balance between scientific output and management and maintenance of large teams of robots and people.

- Failure in balancing the evolution versus revolution threshold over time. Too much forward pressure could lead to teams quitting because they cannot keep up, too little forward pressure could lead to high-ranking teams quitting because the competition is not challenging enough.

- League getting inaccessible for new teams because of high startup effort.

\subsection{Strategy}

The necessarily short analysis presented in the previous subsection can be summarized in a single fundamental question: How are we going to keep making technical and scientific achievements, while also attracting more teams and reducing costs? The answer, in our view, is threefold:

- Increase of knowledge sharing by encouraging scientific publications, release of well established and tested middle-ware and sharing of hardware solutions. A significant effort is already going on in all these areas. Examples include the three day RoboCup MSL workshop held at the University of Kassel last year and the launch of ROP (Robotic Open Platform). ${ }^{3}$ With a wiki, a Q\&A section and a CAD file repository, ROP facilitates the release of hardware designs of robots and modules under an open hardware license. MSL robots of team Tech United have been fully released already. A release of the CAMBADA robot, among others, is currently being prepared.

- Design and production of an affordable robot platform for MSL, able to provide a starting point for any new team. A robot that plays basic soccer out of the box and allows new teams to literally build on knowledge that is already accumulated in the league. With this goal in mind, the TURTLE$5 \mathrm{k}$ consortium was initiated shortly after RoboCup Mexico. ${ }^{4}$ At the time of writing a first team of robots has been sold and completed.

- Discussion and annual evaluation of the MSL roadmap, targeting objectives for the short, medium and long run and using the Technical Challenge to prepare for upcoming rule or environment changes.

${ }^{3}$ ROP, An online platform for Open Hardware releases, initiated by Eindhoven University of Technology, http://www.roboticopenplatform.org/.

${ }^{4}$ TURTLE-5k Consortium: ACE, VEDS, Frencken and Eindhoven University of Technology, http://www.turtle5k.org/. 


\section{The Future}

MSL owns an important piece in solving the RoboCup challenge. It continuously gets fed by the simulation, standard platform and small-size leagues and, in turn, knowledge accumulated at MSL finds its way to other leagues. ${ }^{5}$ Eventually, the RoboCup Challenge is formulated in terms of a humanoid team. Therefore, as soon as MSL is able to build a team of non-humanoid robots able to beat a human team, while playing by the FIFA rules on an official turf, close cooperation with the humanoid leagues should be started. It is not unthinkable, by many it is even expected, that around 2025 the MSL has evolved into a legged league as well, ${ }^{6}$ although this will not be enforced by the rules.

In the short run we are focussing on other things. Further improving and maintaining the $5 \mathrm{k}$ platform is crucial for the league, as well as adding more robot designs to ROP. In order to better present ourselves to universities and companies potentially interested in joining, we are working on a remake of the MSL website. The new site will have search functionality and tagging to browse trough a list of publications related to MSL.

The artificial grass challenge during RoboCup Brazil has the potential to be revolutional. Once teams manage to drive, dribble, pass and shoot on artificial grass, we can speak of a new epoch in MSL. The different characteristic of artificial grass in comparison to carpet will have their effect on base movement and base stability. Probably it will also influence robot vision, which has to deal with much more tilt and vibrations, while active ball handling systems will have to be redesigned to deal with the reduced friction. One of the teams has announced to introduce a fully suspended soccer robot during the technical challenge in Brazil, which would be unique within MSL.

Also new in Brazil will be standardized logging of game data. Via the refbox pc, at a fixed sample rate, teams will fill in a struct with parameters like, e.g., the estimated location of the ball and a list of candidate obstacles. Once further extended, publishing of such a standardized struct can ease up mixed team soccer playing. Data will also be put online, enabling post game analysis or application of machine learning techniques to robot soccer.

The decision to log data and other plans presented in this paper were made during the 2013 RoboCup MSL workshop in Kassel. With this years workshop planned to take place in Eindhoven, and the one next year already planned in Aveiro it will be an annual event where proposed rule changes are discussed and teams obtain hands-on experience in working with each others development environment and toolchain.

Lastly, although continuously under discussion, we present snippets of some important subjects on our roadmap (Table 1 ).

\footnotetext{
${ }^{5}$ The robots of RoboCup@Home teams CAMBADA and Tech United Eindhoven are largely based on the soccer robots made by those teams.

${ }^{6}$ Already in 2010 a first prototype of a hexapod robot for MSL was presented during the scientific challenge. This project is still ongoing.
} 
Table 1. Part of the proposed roadmap for in-game changes and technical challenges (TC). Subject to yearly review.

\begin{tabular}{c|l|l|l|l}
\hline & 2014 & 2015 & 2016 & - \\
\hline Ground & $\begin{array}{c}\text { Artificial grass } \\
\text { (TC) }\end{array}$ & $\begin{array}{c}\text { Artificial grass } \\
\text { (demo match) }\end{array}$ & $\begin{array}{c}\text { Artificial grass } \\
\text { (competition) }\end{array}$ & $\begin{array}{c}\text { Real grass } \\
\text { outdoors (TC) }\end{array}$ \\
\hline Passing & $\begin{array}{c}\text { One pass before } \\
\text { scoring }\end{array}$ & $\begin{array}{c}\text { One pass before } \\
\text { scoring, ex. rest. }\end{array}$ & $\begin{array}{c}\text { Two passes } \\
\text { before scoring }\end{array}$ & $\begin{array}{c}\text { Stimulate } \\
\text { in-game lob pass }\end{array}$ \\
\hline Dribbling & $\begin{array}{c}\text { Circle max } 3 \mathrm{~m} \\
\text { radius }\end{array}$ & $\begin{array}{c}\text { Circle max } 2 \mathrm{~m} \\
\text { radius }\end{array}$ & $\begin{array}{c}\text { Circle max } 1 \mathrm{~m} \\
\text { radius }\end{array}$ & $\begin{array}{c}\text { Limit } \\
\text { ball-holding time }\end{array}$ \\
\hline seam & $\begin{array}{c}\text { Cyan/Magenta } \\
\text { on black }\end{array}$ & $\begin{array}{c}\text { Saturated colors } \\
\text { from predef. set }\end{array}$ & $\begin{array}{c}\text { Random color } \\
\text { opponent (TC) }\end{array}$ & $\begin{array}{c}\text { Free saturated } \\
\text { colors }\end{array}$ \\
\hline $\begin{array}{c}\text { Coaching } \\
\text { QR and color } \\
\text { Mixed }\end{array}$ & $\begin{array}{c}\text { Call robot with } \\
\text { team }\end{array}$ & $\begin{array}{c}\text { Only voice and } \\
\text { geicestures allowed }\end{array}$ & $\begin{array}{c}\text { Directly respond } \\
\text { also to referee }\end{array}$ \\
\hline
\end{tabular}

\section{Conclusions}

RoboCup MSL takes the shortest route to FIFA compliant, but not necessarily humanoid, autonomous robot soccer playing. The annual match of the MSL winner against the trustees is an exposure of the worldwide state-of-the art in human versus robot soccer playing. Epochs of evolution and revolution observed over the past 18 years show that MSL contributed significantly to solving the RoboCup Grand Challenge. With an ambitious roadmap and multiple initiatives to allow new teams to catch up quickly, substantial contributions can also be expected in the years to come.

Acknowledgments. The authors express their deepest gratitude to every team or individual that, since 1997, either at the regional or worldwide level, has contributed to the technical and scientific progression of RoboCup MSL.

\section{References}

More than 450 technical and scientific documents have been produced in relation to RoboCup MSL. On our wiki a pdf and bibtex list is maintained. ${ }^{7}$

\footnotetext{
$\overline{7}$ http://wiki.robocup.org/wiki/Middle_Size_League.
} 